\title{
Utilización y verificación de servicios climáticos para gestión del regadío
}

https://doi.org/10.31978/639-19-010-0.175

\author{
Antonio Ángel Serrano de la Torre ${ }^{1}$ (aserranot@aemet.es) \\ Irene Mestre Guillén' (imestreg@aemet.es) \\ Ernesto Rodríguez Camino1 (erodriguezc@aemet.es) \\ José Antonio López Díaz (jlopezd@aemet.es)
}

${ }^{1}$ AEMET / Dirección de Producción e Infraestructuras

\begin{abstract}
RESUMEN
El proyecto europeo MOSES ${ }^{1}$ (Managing crOp water Saving with Enterprise Services) tiene como objetivo principal contribuir al ahorro de agua mediante la mejora de los servicios a los agricultores y la reducción de costes económicos y energéticos. Para ello, implementa, a nivel de usuarios reales, una plataforma que servirá de ayuda en la gestión del almacenamiento del agua de regadío. AEMET participa en el proyecto MOSES realizando predicciones meteorológicas a corto/medio plazo y estacionales. Dichas predicciones abarcan dos áreas piloto en España, y otra en Marruecos.
\end{abstract}

Las variables proporcionadas por AEMET son, básicamente, temperatura, precipitación y evapotranspiración de referencia, siendo esta última de especial utilidad para la planificación del riego. AEMET ha adaptado algunos productos preexistentes para su utilización en el proyecto MOSES. Esta adaptación incluye la regionalización temporal y espacial de las variables previstas a escala climática. Las variables producidas de este modo están destinadas a alimentar un modelo de balance de agua en el suelo, que es el que proporciona la estimación de las necesidades de riego.

En este trabajo se presentan los datos y métodos utilizados para confeccionar las predicciones meteorológicas en el corto/medio plazo, por un lado, y a escala estacional por otro, así como la verificación de ambos tipos de producto. También se proporciona la verificación de dichas predicciones. Además, estas predicciones se ponen en contexto dentro del objetivo general del proyecto MOSES.

La verificación de los productos indica su idoneidad para el corto/medio plazo y la necesidad de mejorar las predicciones estacionales. En todo caso se hacen algunas propuestas para mejorar ambos tipos de predicciones para aplicaciones agrarias.

PALABRAS CLAVE: aplicaciones de predicción; moses; regadíos; aplicaciones para la agricultura; análogos de series de tiempo.

\section{INTRODUCCIÓN}

El proyecto EU H2020 MOSES (Managing crOp water Saving with Enterprise Services) nace del interés de la Comisión Europea en poner agua de calidad a disposición de los ciudadanos ${ }^{2}$. Concretamente, tiene por objetivo la implementación y la demostración, al nivel de usuarios reales, de una plataforma que servirá

http://www.moses-project.eu

2 http://ec.europa.eu/environment/water/quantity/scarcity_en.htm 
de ayuda a los agentes encargados del almacenamiento y la gestión del agua. Estos utilizarán la plataforma como ayuda a la planificación de los recursos hídricos destinados a regadíos. Dentro de este objetivo, el proyecto contribuye a mejorar aspectos críticos como son los sistemas de monitorización y de toma de decisiones, y políticas de gestión del agua.

Colaboran un total de 16 instituciones entre las que se encuentran agencias ambientales, institutos de investigación, asociaciones relacionadas con la explotación e investigación espacial, consorcios del agua, asociaciones de regantes, pequeñas y medianas empresas, e industrias de 5 países europeos y 3 continentes. Hay además 3 socios colaboradores externos. Mención especial merecen los socios: Universidad Delft (Holanda), Universidad Chouaïb

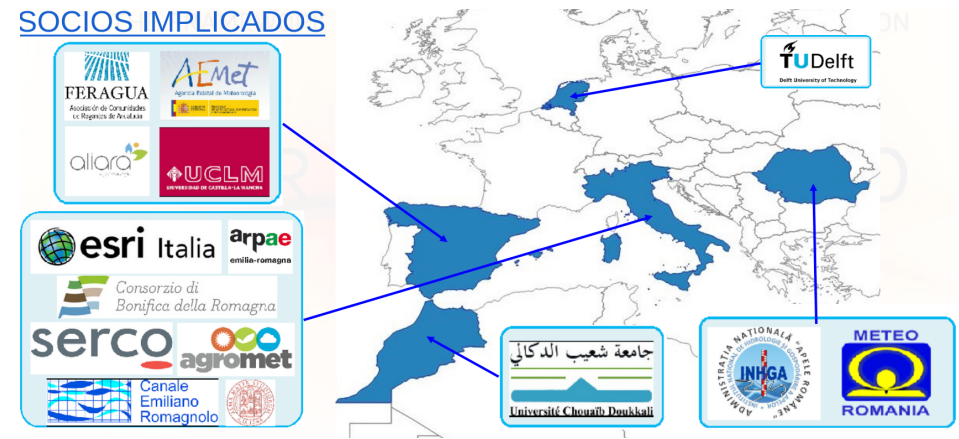

Figura 1. Principales socios implicados en el proyecto MOSES.

Doukkali (Marruecos) y los organismos meteorológicos de España, Italia y Rumanía. Por parte de España, los socios son, además de AEMET: Feragua (Asociación de Comunidades de Regantes de Andalucía), UCLM (Universidad de Castilla - La Mancha, que se ocupa de procesar datos de teledetección) y Aliara Agrícola (empresa de consultoría para agricultores). Por parte de Italia, cabe destacar también a ESRI Italia, que se ocupa de la dirección general del proyecto, y ARAPAE (Agenzia Regionale Prevenzione e Ambiente dell'Emilia Romagna), que realiza predicciones estacionales meteorológicas que cubren todas las áreas piloto del proyecto. ARPAE confecciona estas predicciones a partir de las salidas de los modelos integrantes del proyecto Eurosip ${ }^{3}$. Podemos ver un listado más amplio de participantes en la figura 1.

Los productos se han desarrollado para 4 áreas de piloto que se han escogido atendiendo a su importancia en el sector agrario y que se encuentran localizadas en Italia, España, Rumanía y Marruecos. Los productos desarrollados son: Información Pre-estacional procedente de teledetección, Información Estacional procedente de teledetección y de predicción estacional del tiempo, e Información Intra-estacional procedente de teledetección y pronósticos del tiempo.

La contribución de AEMET consiste en la producción de predicciones meteorológicas en el corto/medio plazo (hasta siete días) y a escala estacional, que cubren dos áreas piloto en España y otra en Marruecos. Las áreas españolas son BembézarMD (circa 12000 ha) y Sector-BXII (circa 15000 ha), que se encuentran en la cuenca del río Guadalquivir. El área marroquí se llama Doukkala (96 000 ha) y está localizada entre las montañas del Atlas y la costa atlántica.

El objetivo de AEMET es proporcionar dos tipos de productos: predicciones a corto/medio plazo y predicciones estacionales. Las predicciones a corto/medio plazo cubren hasta un alcance de 7 días. Las predicciones estacionales cubren una estación de tres meses que comienza en el mes siguiente a aquel en el que se realiza la predicción.

La predicción a corto/medio plazo proporciona las variables: temperaturas mínima, máxima y media, precipitación, evapotranspiración de referencia, humedad relativa mínima, máxima y media, velocidad media del viento y radiación global. La predicción estacional proporciona las variables: precipitación, temperatura mínima y temperatura máxima. En ambos tipos de producto se proporciona dato diario. Debemos hacer notar que ARPAE proporciona predicciones estacionales para todas las áreas piloto usando un método común para todas. Sin embargo, es responsabilidad de cada organismo meteorológico proporcionar también predicciones estacionales para las áreas de su responsabilidad utilizando un método específico. En el presente trabajo nos centramos solamente en el método específico usado por AEMET.

\footnotetext{
https://www.ecmwf.int/en/forecasts/documentation-and-support/long-range/seasonal-forecast-documentation/eurosip-user-guide/multi-model
} 
En este artículo se describen estos dos tipos de productos poniéndolos en contexto dentro del objetivo general del proyecto de proporcionar predicciones de necesidades de riego, y limitándonos a los que se han generado para las áreas piloto españolas.

El citado contexto es, para las predicciones meteorológicas a corto/medio plazo, la predicción a corto/medio plazo de las necesidades de riego; y para las predicciones estacionales, las predicciones estacionales de necesidades de riego. A continuación se hace una breve descripción de estos dos contextos.

\subsection{Predicción de riego a corto/medio plazo}

El elemento central de la predicción de riego, tanto a corto/medio plazo, como estacional, es un modelo de balance de agua en el suelo (o SWB del inglés Soil Water Balance). Concretamente, en este proyecto se ha usado el programa Criteria ${ }^{4}$.

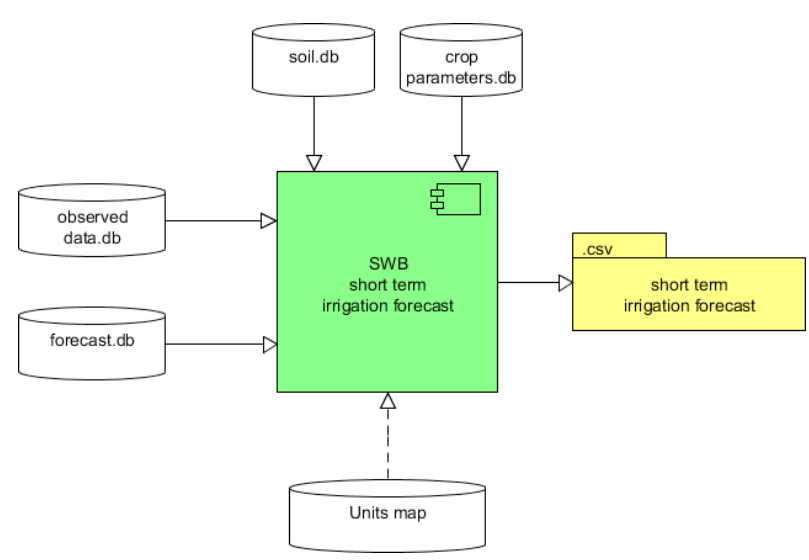

Figura 2. Esquema del cálculo de la predicción a corto/medio de las necesidades de riego. Recibe entrada de la predicción determinista a corto/medio plazo, y de una serie de tiempo diaria de un año de observaciones.

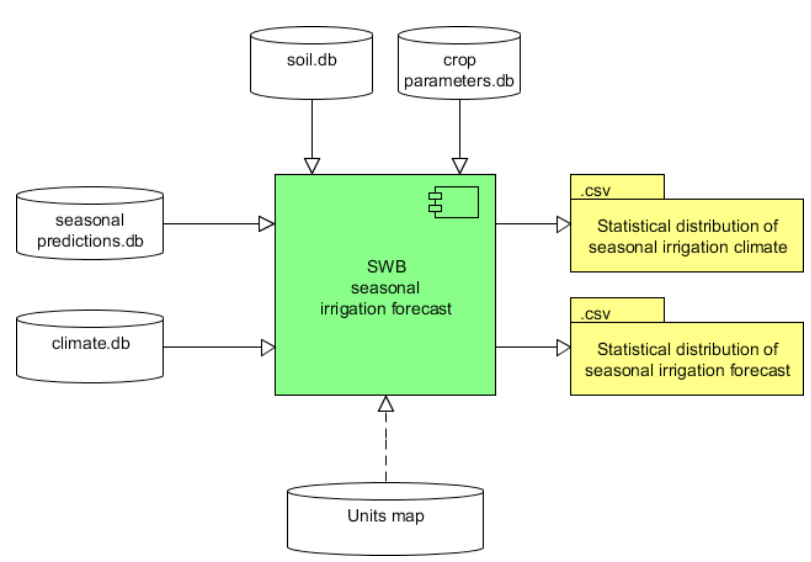

Figura 3. Esquema del cálculo de la predicción estacional de las necesidades de riego. Recibe entradas probabilísticas en modo de ensembles y produce salidas también probabilísticas en modo de ensembles.
En la predicción a corto/medio plazo (fig. 2), los datos de entrada que el modelo de balance necesita son: datos referentes al suelo (composición, capa freática, etc.), parámetros del cultivo (dependientes del tipo de cultivo, su estado de crecimiento, etc.), predicción meteorológica determinista a siete días (valor diario), y una serie de tiempo de observaciones diarias de al menos un año de longitud y que termina el día anterior al primer día de la predicción, formando con esta una serie diaria continua. También recibe información acerca de la estructura espacial de las predicciones meteorológicas, las cuales se dan en los nodos de una malla que debe ser especificada.

El resultado proporcionado por el modelo de balance de agua es una predicción de riego para los próximos 7 días.

\subsection{Predicción estacional de riego}

El esquema de la predicción estacional de riego (fig. 3) es bastante similar al correspondiente a la predicción a corto/medio plazo. Al igual que entonces, también recibe como entrada las características del suelo. En cuanto a las características de los cultivos, se obtienen de un promedio hecho en un periodo «climatológico».

Los datos meteorológicos que recibe son una predicción meteorológica estacional y una climatología. Ambos datos deben darse como un conjunto o ensemble de series de tiempo diarias de temperatura y precipitación. Por último, el modelo de balance de agua también recibe información acerca de la estructura espacial de las predicciones meteorológicas, las cuales se dan en los nodos de una malla que debe ser especificada.

\footnotetext{
${ }_{4}$ Para más información sobre el modelo de balance de agua Criteria, puede verse: http://moses-project.eu/moses_website/criteria-model/
} 
El modelo proporciona dos resultados. Por un lado, la predicción estacional de las necesidades de riego. Se trata de una predicción probabilista que se produce como un ensemble de series de tiempo diarias. Por otro lado, también proporciona la «climatología» de las necesidades de riego, es decir, las necesidades de riego predichas mediante las estadísticas en un periodo de «climatología» y no mediante la predicción meteorológica estacional. Es también un ensemble de series de tiempo diarias.

\section{DATOS Y MÉTODOS}

\subsection{Datos y métodos para la predicción a corto/medio plazo}

La predicción a corto/medio plazo se basa en la base de datos digital de predicción (de ahora en adelante, BDDP). Este es un producto interno destinado a ayudar a los predictores en su trabajo rutinario. Está formado, para alcances dentro del corto plazo, por la salida de modelos dinámicos regionales como Hirlam o Harmonie, y para alcances en el medio plazo, por el modelo determinista del Centro Europeo. Estas salidas de modelos han sido mejoradas mediante un postproceso antes de pasar a formar parte de la BDDP. Dicho postproceso utiliza datos observacionales. Tanto la resolución espacial como la temporal de la BDDP, es apropiada para nuestros propósitos, de modo que no es necesaria ninguna regionalización temporal ni espacial.

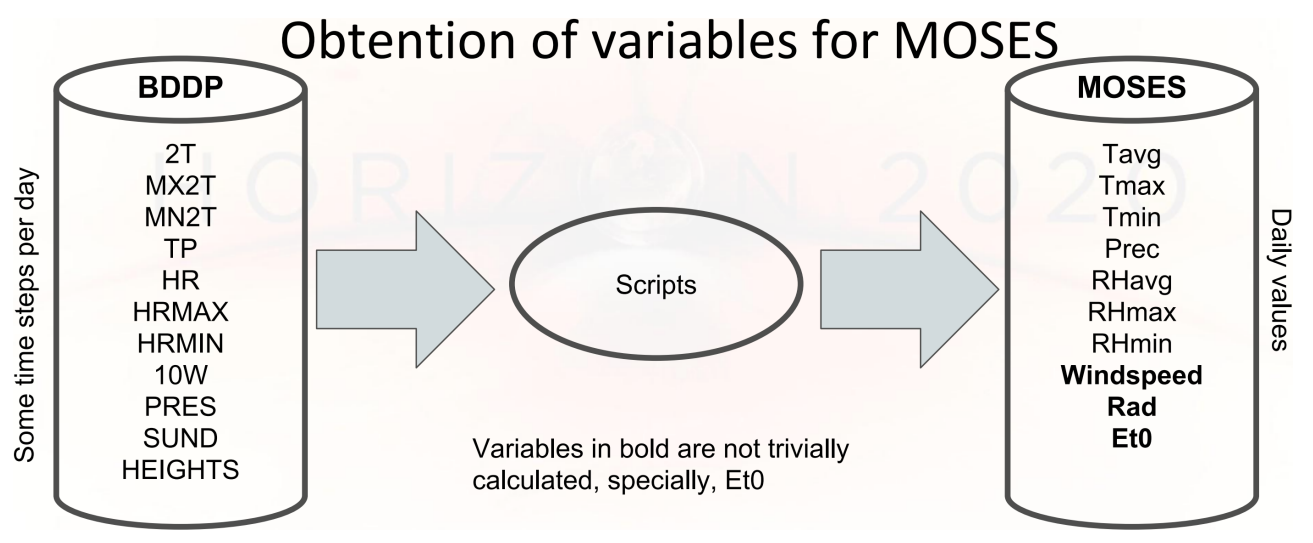

Figura 4. Variables existentes en la BDDP y variables necesarias para el proyecto MOSES (corto/medio plazo).

Las variables necesarias para el proyecto MOSES, se calculan a partir de las proporcionadas por la BDDP (fig. 4). La insolación (a partir de la cual se calcula la radiación global) se obtiene del modelo determinista del Centro Europeo para todos los alcances, ya que esta variable no está incluida en la BDDP. El cálculo de la evapotranspiración de referencia se hace según el método FAO Penman-Monteith, ligeramente adaptado al uso de datos de predicción en vez de datos de observación, que es lo que asume el método. De este modo, mientras la FAO recomienda que el valor medio diario de diversas variables se calcule usando sus valores extremos (máximo y mínimo) en este trabajo se ha usado el promedio de cuatro valores diarios (separados hexahorariamente). Este producto se genera diariamente y se entrega al proyecto vía FTP.

\subsection{Datos y métodos para la predicción estacional}

La predicción estacional es probabilista y se proporciona como un ensemble de predicciones. Cada miembro del ensemble consiste en dos series de tiempo diarias físicamente consistentes entre sí: una para la temperatura y otra para la precipitación. Está basada en la predicción estacional operativa de AEMET, que se produce mensualmente para la estación compuesta por los tres meses siguientes al mes en el que se produce. 


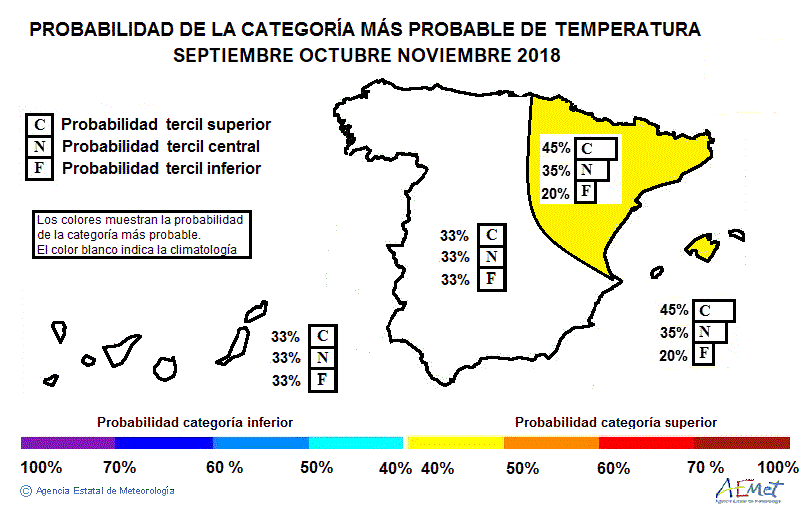

Figura 5. Predicción estacional operativa de AEMET. Se produce una predicción por terciles para cada cuadrante de la Península y para Baleares y Canarias.
La predicción estacional operativa se hace por consenso usando una selección de modelos climáticos e impulsores del clima relevantes a escala estacional. Se divide el territorio nacional en zonas para las que se prevé un comportamiento homogéneo y se asigna una predicción por terciles a cada una de ellas. Podemos ver un ejemplo de esta predicción en la figura 5.

Esta predicción se regionaliza espacial y temporalmente mediante el método de los análogos de series de tiempo, que produce un conjunto de series de tiempo diarias de temperatura y precipitación. Este método es más simple que otros que también se usan en AEMET y permite el uso inmediato de la predicción estacional operativa. Además, hace innecesaria la aplicación de un generador de tiempo para producir datos de precipitación y temperatura físicamente consistentes. Sin embargo, su principal inconveniente es que el número de miembros (pares de series de tiempo temperaturaprecipitación) que produce es variable, normalmente entre 15 y 40 para cada variable meteorológica.

El método de los análogos de series de tiempo consiste en la selección de pares de series de tiempo diarias de temperatura-precipitación pertenecientes a la estación del año a predecir, a partir de series de tiempo de observaciones de una estación meteorológica. De este modo, partiendo de un número de pares de series de tiempo de la estación del año requerida, igual al número de años de observaciones, procedemos a clasificar cada año como perteneciente a un determinado tercil de temperatura y a un determinado tercil de precipitación. Lo que pretendemos entonces es seleccionar un número de miembros (pares de series de tiempo temperatura-precipitación) de modo que su distribución en los terciles de temperatura y precipitación sea lo más cercana posible a las probabilidades proporcionadas por la predicción estacional operativa. Los terciles, por otra parte, se definen usando la misma climatología que la usada por la predicción estacional operativa.

Supongamos por ejemplo que disponemos de 55 años de observaciones diarias y que la predicción para la temperatura del próximo invierno es de $33 \%, 33 \%, 33 \%$ (por debajo de lo normal, normal y por encima de lo normal), y para la precipitación es $25 \%, 35 \%, 40 \%$. Si el observatorio no está muy lejos del lugar para el que queremos hacer la regionalización de la predicción, podemos considerar que el tiempo meteorológico es el mismo en ambos lugares.

En cada uno de los años de observación, seleccionamos los días que pertenecen a la estación del año a predecir, en este ejemplo, invierno. Después se calcula la temperatura media y la precipitación acumulada de esos días seleccionados, en cada año. Dicha temperatura media caerá en un determinado tercil de temperatura, y la precipitación acumulada, en otro determinado tercil de precipitación.

Hay un total de nueve posibilidades para clasificar un año como perteneciente a una determinada combinación de terciles de temperatura y precipitación. Podemos ver dichas posibilidades en la tabla 1 , donde T1, T2 y T3 designan al primer, segundo y tercer terciles de temperatura, respectivamente, $\mathrm{y}$ P1, P2 y P3 designan al primer, segundo y tercer terciles de precipitación, respectivamente. Las frecuencias absolutas y relativas de ocupación de la precipitación y la temperatura se encuentran en las dos últimas filas y en las dos últimas columnas, respectivamente.

\begin{tabular}{|l|ccccc|}
\cline { 2 - 6 } \multicolumn{1}{c|}{} & T1 & T2 & T3 & Freq. abs. Freq. rel. \\
\hline P1 & 6 & 6 & 2 & 14 & $25 \%$ \\
P2 & 9 & 9 & 1 & 19 & $35 \%$ \\
P3 & 2 & 8 & 12 & 22 & $40 \%$ \\
Freq. abs. & 17 & 23 & 15 & & \\
Freq. rel. & $31 \%$ & $42 \%$ & $27 \%$ & & \\
\hline
\end{tabular}

Tabla 1. Frecuencias de ocupación de los miembros observacionales. 
El objetivo ahora es seleccionar un número de años del total de 55 de la tabla 1, de modo que las frecuencias relativas de temperatura y precipitación sean lo más cercanas posible a las probabilidades proporcionadas por la predicción estacional.

Para solucionar este problema de optimización restringida, se ha elaborado un algoritmo de búsqueda exhaustiva que, para el número de años disponibles en este estudio, es perfectamente viable y se ejecuta en un tiempo razonable. En primer lugar, se define una métrica para cada tabla posible de números de ocupación. Dicha métrica mide la distancia al objetivo deseado. Para definirla, partimos del vector de seis elementos formados por la concatenación de las frecuencias relativas de los terciles de temperatura y precipitación (última fila y última columna de la tabla 1). Después se calcula la suma de los cuadrados de los elementos de este vector. El número obtenido es el valor de la métrica (se trata de una métrica euclídea).

De este modo, se comienza con el vector de frecuencias relativas mostrado en la tabla 1: $(31,42,27,25,35,40)$. Después se seleccionan algunos, o ninguno, de los años de observación en cada celda intersección de los terciles de temperatura y precipitación. Esta selección se hace de manera que se obtenga un vector de frecuencias relativas igual a, o lo más aproximado a, el vector de probabilidades de la predicción: $(33,33,33,25,35,40)$.

Sean $i=\mathrm{P} 1, \mathrm{P} 2, \mathrm{P} 3$ y $j=\mathrm{T} 1, \mathrm{~T} 2, \mathrm{~T} 3$. Sea también $\mathrm{f}_{i j}$ la frecuencia absoluta de la celda $i, j$. Para $i=\mathrm{P} 1 \mathrm{y}$ $j=\mathrm{T} 1$ se tiene un conjunto de 6 años, de modo que $\mathrm{f}_{\mathrm{P} 1, \mathrm{~T} 1}=6$, y se pueden elegir, de esta celda, $0,1 \ldots$ o 6 años para hacer que las frecuencias relativas sean lo más parecidas posible a las probabilidades de la predicción. De este modo, de cada celda se puede escoger uno cualquiera de entre $\left(\mathrm{f}_{i j}+1\right)$ subconjuntos. Por tanto, el número total de subconjuntos de todas las celdas es $\Pi\left(\mathrm{f}_{i j}+1\right)$ donde el producto se extiende a las 9 celdas de ocupación de la tabla 1. En el ejemplo ilustrado, este número es del orden de $10^{7}$.

De todas las combinaciones posibles, se escoge aquella que minimiza la métrica (véase tabla 2). Si el número de años fuese considerablemente mayor, el número total de combinaciones crecería exponencialmente, por lo que sería más adecuado el uso de la técnica de Monte Carlo.

Comparando las tablas 1 y 2 podemos ver, por ejemplo, que en la celda (P1,T1), teníamos 6 años, de los cuales hemos seleccionado solo 4. No importa cuáles de los 6 años iniciales se hayan escogido, ya que cualquier opción llevaría al

\begin{tabular}{|c|ccc|}
\cline { 2 - 4 } \multicolumn{1}{c|}{} & T1 & T2 & T3 \\
\hline P1 & 4 & 5 & 2 \\
P2 & 9 & 6 & 1 \\
P3 & 2 & 4 & 12 \\
\hline
\end{tabular}

Tabla 2. Números de ocupación optimizados. mismo vector de frecuencias. Sin embargo, también es cierto que, si aplicamos un criterio, estaremos tomando una decisión acerca de los datos. Por ejemplo, si seleccionamos, de entre los 6 años disponibles, los primeros 4, estos estarán menos afectados por el cambio climático que los últimos 4, de modo que la misma temperatura media se alcanza mediante más olas de calor que en los últimos 4 años. Esto proporcionaría unas series de tiempo de temperatura con una estructura muy diferente de la que tendrían los últimos 4 años. Esto puede ser deseable o no, dependiendo del problema que queramos tratar. En este estudio se ha decidido no tomar ninguna decisión acerca de los datos y, por tanto, se han elegido los 4 años de un modo aleatorio.

En la tabla 3 se muestran las frecuencias de ocupación de: el objetivo (la predicción estacional), inicial de la tabla 1 y final de la tabla 2. El algoritmo consiguió una reducción considerable del valor de la métrica, desde 11 para la matriz inicial, hasta 1,4 para la matriz final.

Por último, se toma cada serie de tiempo de cada año (temperatura y precipitación) como un miembro

\begin{tabular}{|l|ccc|ccc|}
\cline { 2 - 7 } \multicolumn{1}{c|}{} & \multicolumn{3}{c|}{ Terciles Prec (\%) } & \multicolumn{3}{c|}{ Terciles Temp (\%) } \\
\hline Objetivo & 25 & 35 & 40 & 33 & 33 & 33 \\
Inicial & 25 & 35 & 40 & 31 & 42 & 27 \\
Final & 24 & 36 & 40 & 33 & 33 & 33 \\
\hline
\end{tabular}

Tabla 3. Frecuencias relativas de ocupación del objetivo (predicción), tentativa inicial y resultado final (optimizadas). del ensemble de la predicción. Por construcción, cada una de estas series de tiempo es físicamente consistente entre temperatura y precipitación, de modo que no es necesaria la aplicación de un generador de tiempo.

Estas series de tiempo se usan como entrada de un modelo de balance de agua en el suelo, que calcula las necesidades de irrigación. 


\section{RESULTADOS}

\subsection{Verificación de la predicción a corto/medio plazo}

Todas las variables de la predicción a corto/medio plazo, se han verificado mediante el cálculo de tres índices de verificación que comparan predicciones con observaciones en un periodo que abarca desde 2017-01-07 hasta 2018-08-31. Este es el periodo en el que se han producido las predicciones para el proyecto. Los citados índices de verificación son: el coeficiente de correlación, la raíz del error cuadrático medio (RMSE, por sus siglas en inglés) y el bias o sesgo. Dichas verificaciones se presentan en las figuras 6, 7 y 8 .

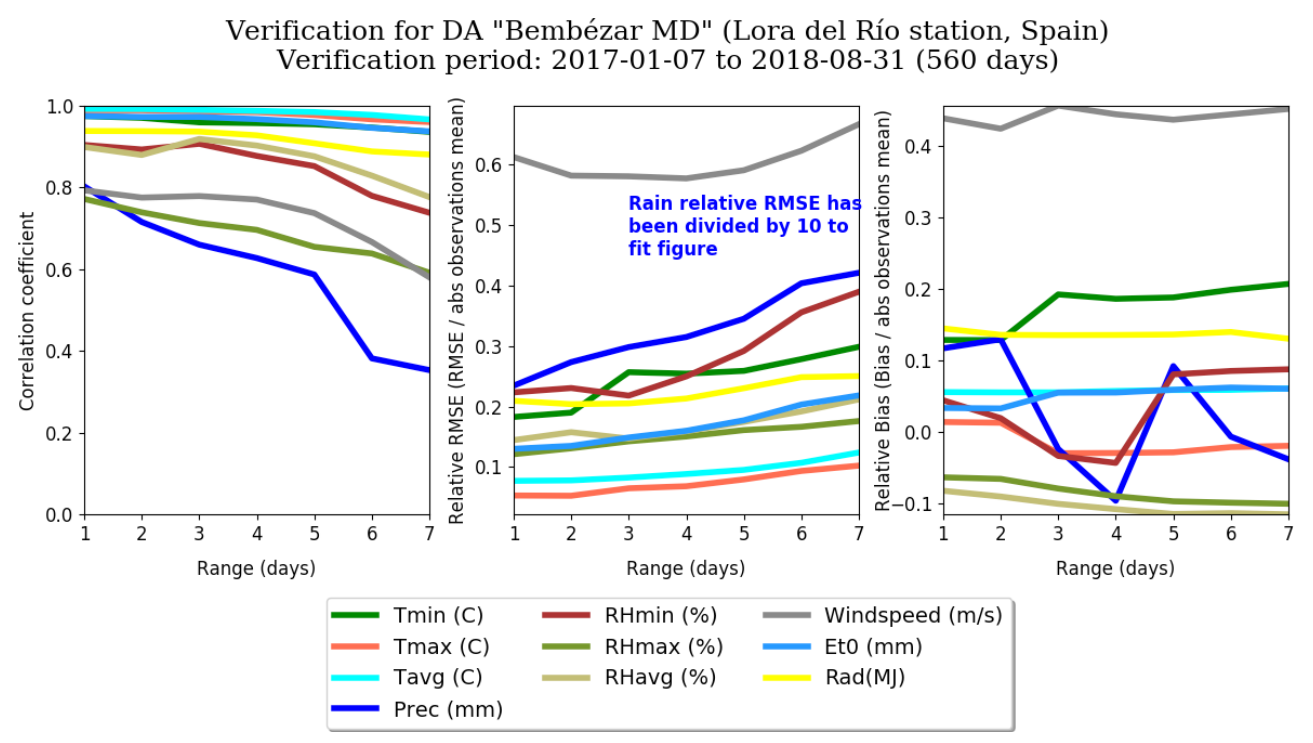

Figura 6. Verificación de las variables generadas en la predicción a corto/medio plazo (7 días). En el eje X, el día que se predice. El 1 corresponde al mismo día en que se lanza la predicción, el 2 al día siguiente, etc. El gráfico de la izquierda representa el coeficiente de correlación entre la predicción a 1, 2..., 7 días y su correspondiente observación. El gráfico central es la raíz del error cuadrático medio dividido entre el valor absoluto de la media de las observaciones para poder representar todas las variables en el

mismo gráfico (además, la curva de la lluvia ha sido dividida entre 10 para que pueda aparecer en el gráfico). El gráfico de la derecha es el sesgo dividido entre el valor absoluto de la media de las observaciones, también, para representar juntas todas las variables.

RMSE for DA "Bembézar MD" (Lora del Río station, Spain) Verification period: 2017-01-07 to 2018-08-31 (560 days)
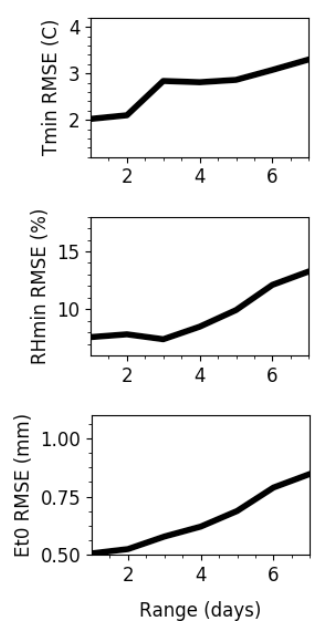
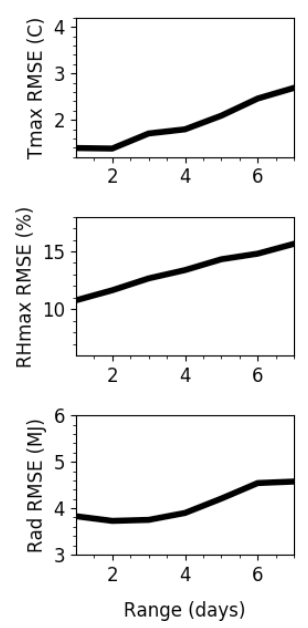
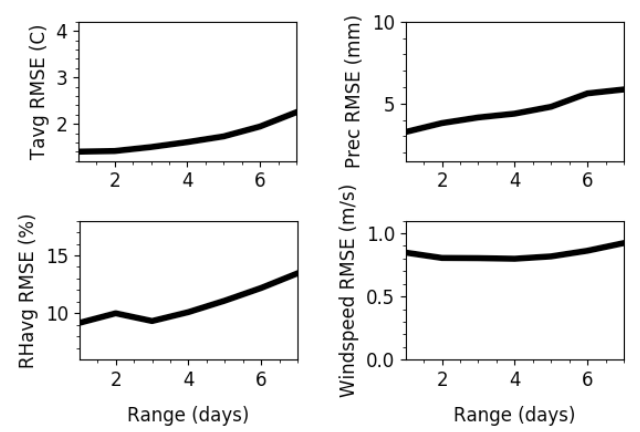

Figura 7. Raíz del error cuadrático medio de cada una de las variables.

Se representa en función del alcance o día que se predice. 
Bias for DA "Bembézar MD" (Lora del Río station, Spain)

Verification period: 2017-01-07 to 2018-08-31 (560 days)
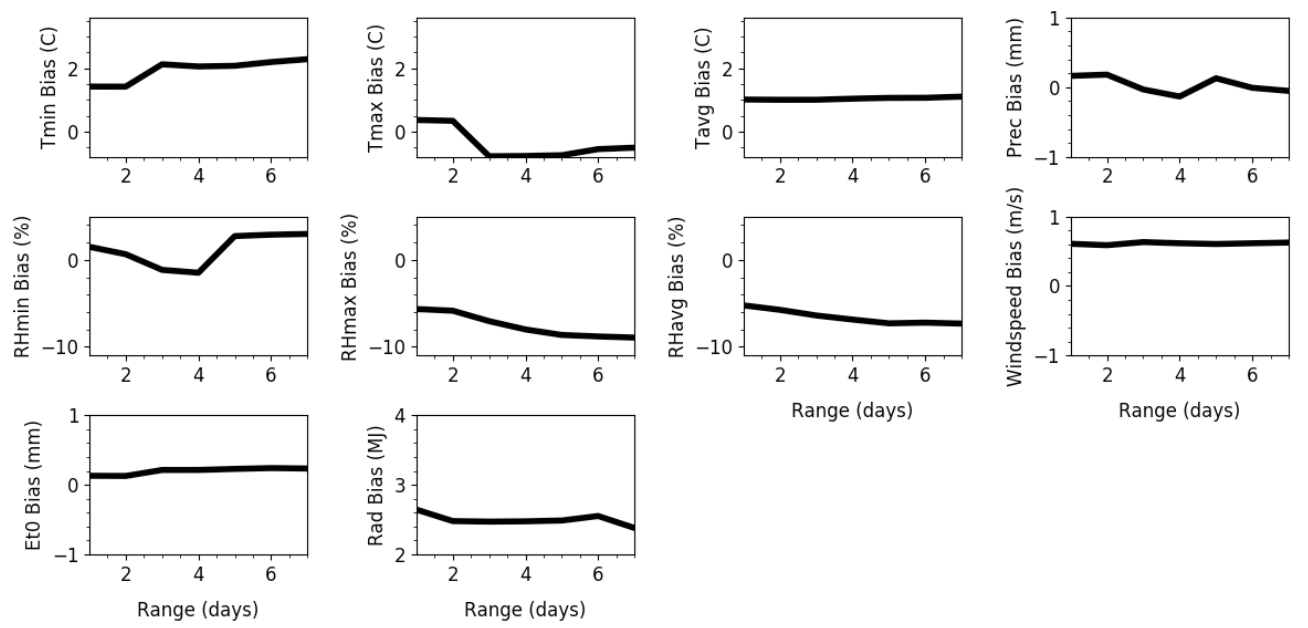

Figura 8. Sesgo de las predicciones de cada una de las variables.

Se representa en función del alcance o día que se predice.

Se observa en las citadas figuras que:

- Todas las variables presentan una buena correlación en los primeros dos días $(\geq 0,7)$.

- Algunas variables presentan una correlación muy buena $(\geq 0,9)$ en los siete días: Et0, Rad, Tmin, Tmax, Tavg.

- Las demás variables se degradan apreciablemente a partir del tercer día, consecuencia del cambio de modelo subyacente.

- Los sesgos son casi constantes para todas las variables. Algunas de ellas presentan un salto que refleja el cambio de modelo subyacente.

- La raíz del error cuadrático medio (RMSE), en general, crece con el alcance siendo casi constante para la velocidad del viento y la radiación.

\subsection{Verificación de la predicción estacional}

Para verificar las predicciones estacionales, debemos tener en cuenta que estas se regionalizan a nivel de estación, es decir, usando observaciones de una estación meteorológica. Esta se escoge de modo que esté suficientemente cercana al área piloto. Recordemos que en el caso estacional, la predicción consiste en un ensemble de series de tiempo de temperatura y precipitación. Dichas series pertenecen a cada tercil en la misma proporción que las probabilidades de la predicción. Por tanto, la verificación de este ensemble equivale a la verificación de las probabilidades de la predicción, es decir, a la verificación de la predicción estacional operativa.

Sin embargo, nos encontramos con el problema de que el número de predicciones operativas producidas hasta el momento es muy pequeño. No hay suficientes casos como para calcular los índices de verificación probabilísticos. Por ello, no es posible una verificación directa. Aun así, se pueden llevar a cabo dos tipos de verificaciones que nos pueden dar una clara indicación de la calidad de la predicción estacional:

1. Verificar la predicción con estaciones de todo un cuadrante peninsular, sin limitarnos a la más cercana al área piloto (comunicación interna).

2. Verificaciones independientes de los principales modelos utilizados para producir la predicción estacional operativa (SÁNCHEZ et al., 2017). Estas se han hecho en toda el área peninsular y en un periodo de hindcast común a todos los modelos (1997-2009). 
Las figuras 9 y 10 muestran los resultados de la verificación de las predicciones estacionales. Solo se presenta la verificación de la lluvia. La temperatura verifica mejor.
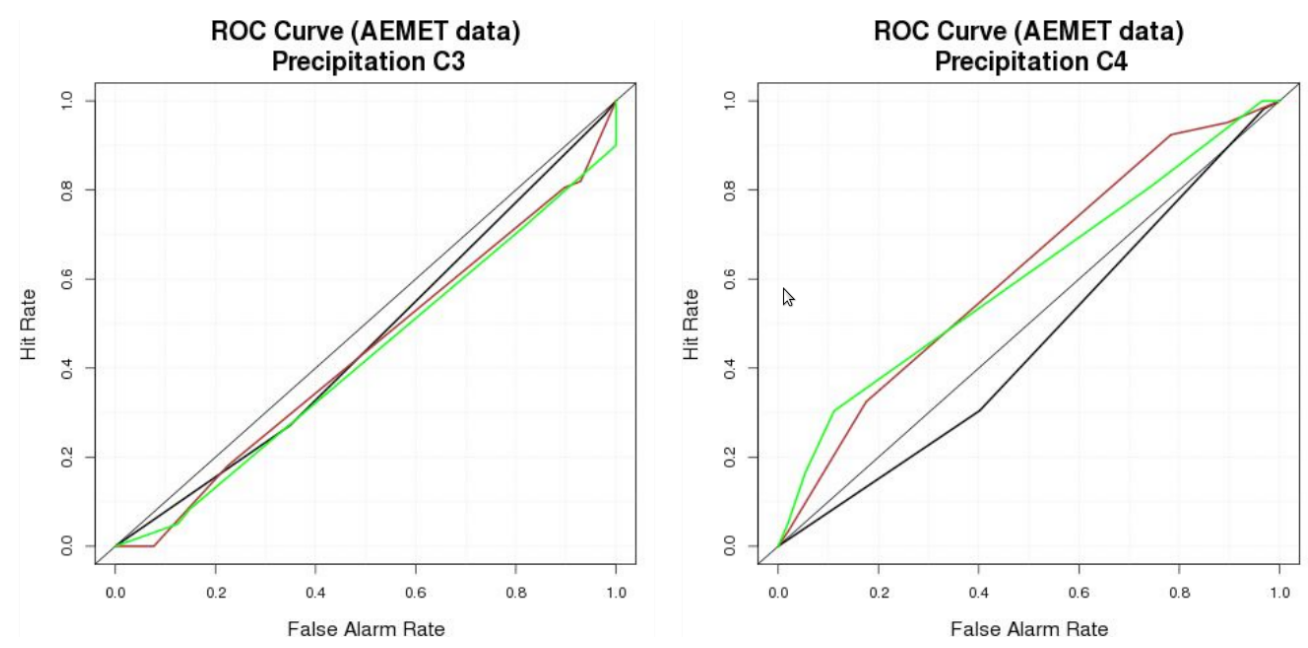

Clave:

Verde: Tercil superior Negro: Tercil medio Marrón: Tercil inferior

Figura 9. Curvas ROC de los cuadrantes sudoeste (a la izquierda, que corresponde al área piloto Sector B-XII) y sudeste (a la derecha, que corresponde al área piloto Bembézar MD).

En las curvas ROC (fig. 9) confeccionadas según el primer tipo de verificación, se puede observar que:

- En el cuadrante sudeste (Bembézar MD) hay alta discriminación para los terciles superior e inferior con alta y baja probabilidades, mientras que no hay discriminación para el tercil central.

- En el cuadrante sudoeste (Sector B-XII) hay una discriminación similar a la de la climatología para todos los terciles.

En cuanto al segundo tipo de verificación, se han verificado los siguientes modelos: European Centre for Medium-Range Weather Forecasts (ECMWF) system 4, Météo-France system 5, UK Met Office system 9 (GloSea5), National Center for Environmental Prediction (NCEP) system version 2, Canadian Seasonal to Inter-annual Prediction System (CanSIPS), Japanese Seasonal Forecasting System 2. Para ello, se ha usado una malla común de $1^{\circ} \times 1^{\circ}$ y un periodo de hindcast común: 1997-2009. Como observaciones se ha usado el conjunto de observaciones interpoladas a rejilla regular Global Precipitation Climatology Centre/Deutscher Wetterdienst (GPCC/DWD).

En este segundo tipo de verificación se observa que hay:

- Mayor capacidad predictiva en general, para la temperatura que para la precipitación (los gráficos para temperatura no se muestran).

- Una relativa consistencia de unos modelos con otros que permite identificar algunas ventanas de oportunidad para predicciones estacionales asociadas a ciertas estaciones meteorológicas, variables y, en algunos casos, limitadas a ciertos modelos.

- Algunos índices de verificación indican más capacidad predictiva que otros, p. ej., el área ROC — que indica la capacidad de discriminación— - tiende a mostrar más capacidad predictiva (respecto de la climatología) que otros índices que exploran otros aspectos de la predicción.

- Para el caso de la precipitación en la península ibérica, algunos modelos tienen resolución para otoño e incluso para invierno. 


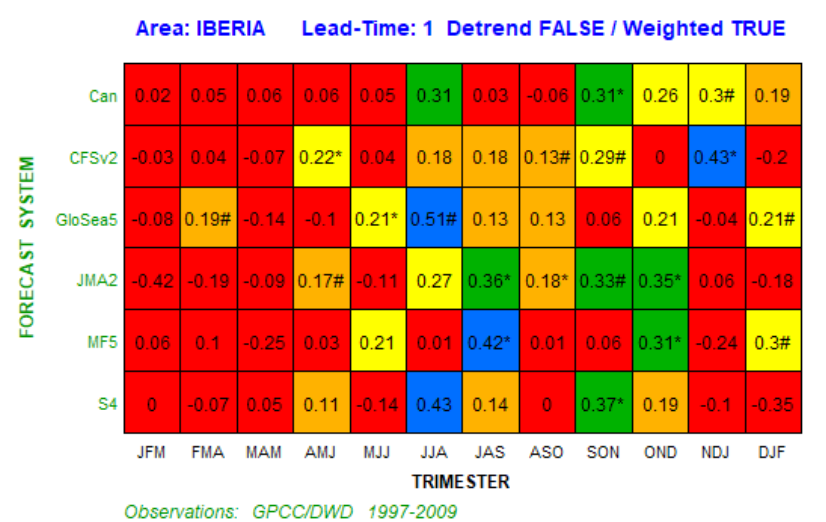

Ooservations. GPCCIDWD 1997-2009

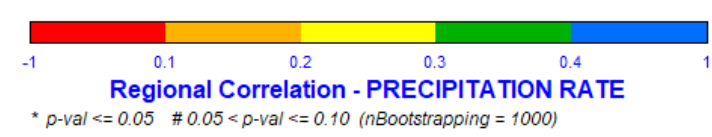

${ }^{*} p$-val $<=0.05 \# 0.05<p$-val $<=0.10$ (nBootstrapping $=1000$ )

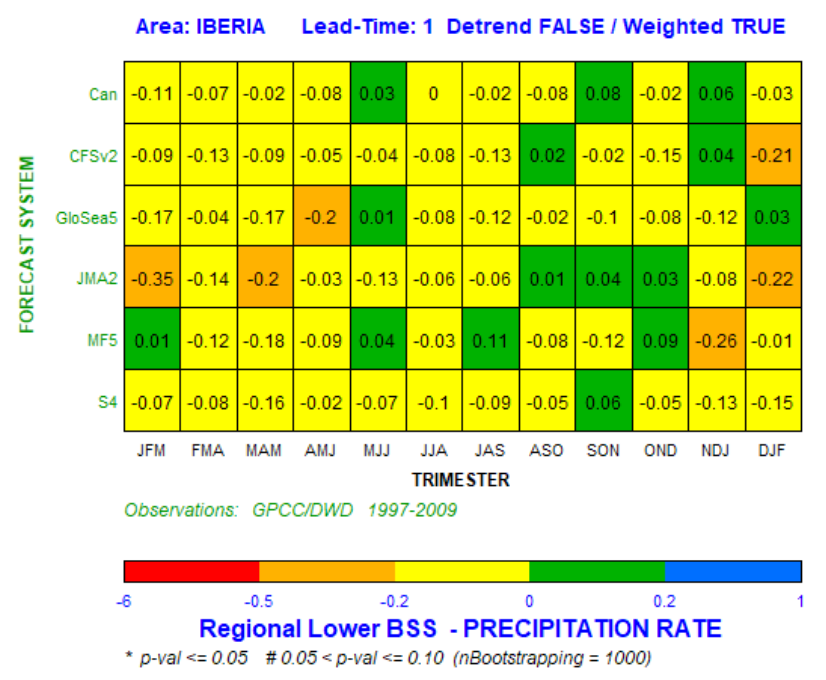

Area: IBERIA Lead-Time: 1 Detrend FALSE / Weighted TRUE

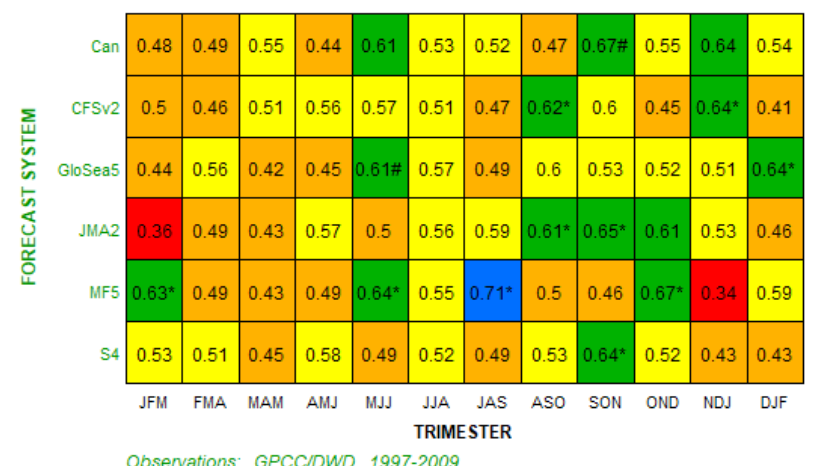

Observations: GPCC/DWD 1997-2009

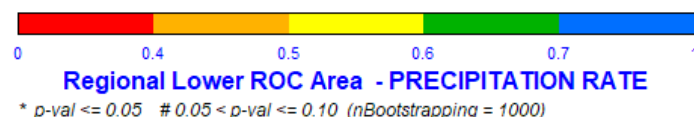

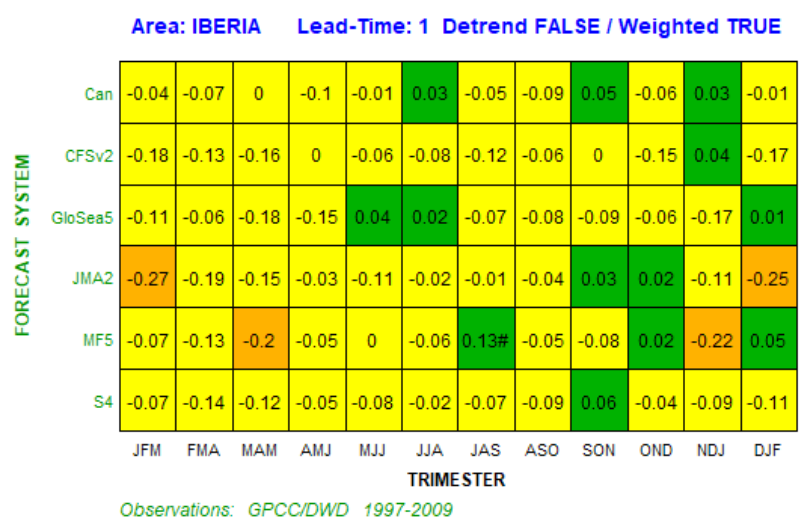
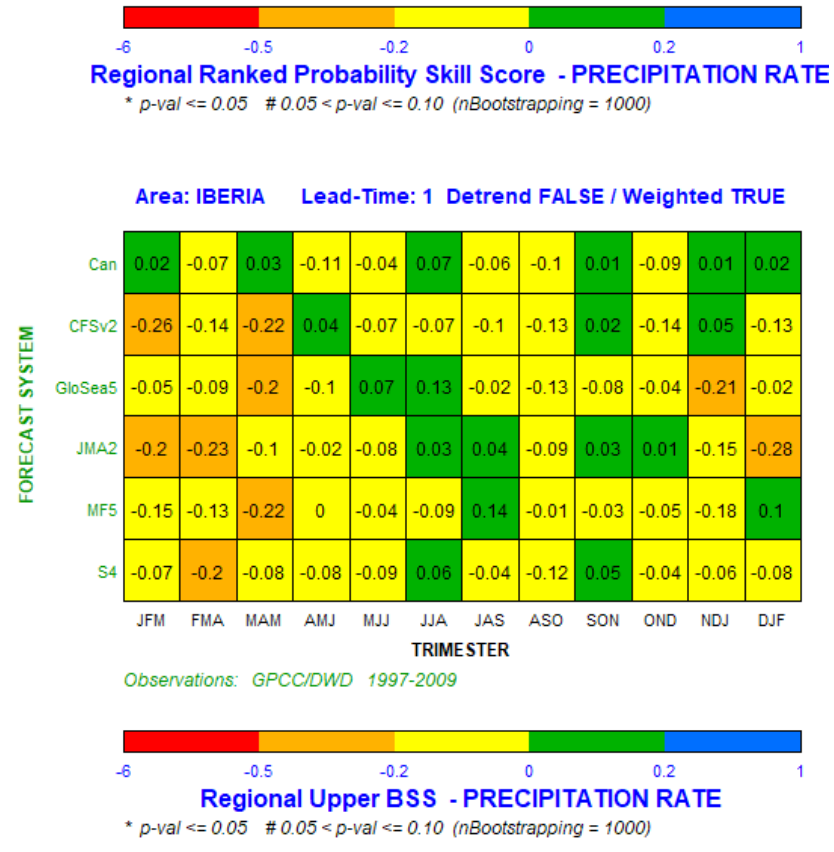

Area: IBERIA Lead-Time: 1 Detrend FALSE / Weighted TRUE

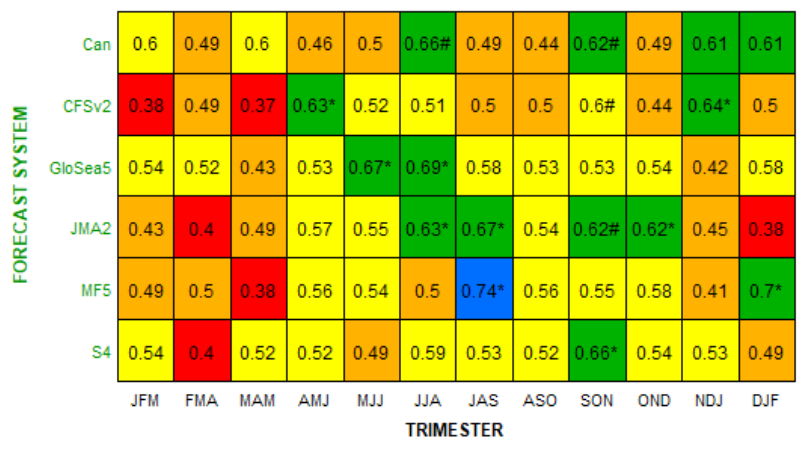

Observations: GPCCIDWD 1997-2009

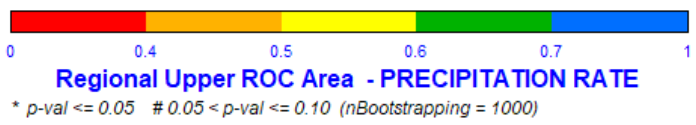

Figura 10. Coeficiente de correlación, Ranked Probability Skill Score, área ROC de los terciles inferior y superior y BSS (Brier Skill Score) calculados para la anomalía de la tempertura, para las doce estaciones de tres meses y para alcance 1. El área geográfica es la península ibérica y el periodo de verificación es 1997-2009. Los periodos de tres meses para los que se produce la predicción estacional se muestran en el

eje X. El modelo sobre el que se hace la verificación (S4, MF5, JMA2, GLOSEA5, CFSv2 y Can) aparece en el eje Y. 


\section{DISCUSIÓN}

\subsection{Discusión de la predicción a corto/medio plazo}

Para cumplir el objetivo principal del proyecto, que es la predicción de necesidades de riego, las variables más importantes son la evapotranspiración de referencia (Et0) y la precipitación.

Las predicciones de Et0 presentan alta correlación, sesgo pequeño $(0<\operatorname{Sesgo}<0,5)$, RMSE pequeño $(<1 \mathrm{~mm}$ en todos los alcances y $<0,6$ para los dos primeros días). Sin embargo, para aplicaciones agroclimáticas sería de gran utilidad poder disminuir aún más ese sesgo. La disminución del sesgo de la Et0 podría conseguirse utilizando el método recomendado por la FAO para el cálculo de los valores medios diarios, es decir, utilizando los valores extremos diarios para calcular la media, en vez de usar 4 valores diarios separados hexahorariamente. Esto sería posible debido a que la predicción de los valores extremos ha venido mejorando bastante debido al avance de los modelos dinámicos y de las técnicas de postproceso aplicadas a las salidas de los mismos.

Las predicciones de lluvia, si bien son bastante buenas en los 2 primeros días, degradan después muy rápidamente. Esto es debido sobre todo al cambio subyacente del modelo, que es regional para el corto plazo y global para el segundo. La aplicación de técnicas de regionalización al modelo global, mejoraría sin duda estos resultados.

\subsection{Discusión de la predicción a corto/medio plazo}

La principal conclusión que se puede obtener de este segundo tipo de verificación es que, en general, esta muestra, como era de esperar, poca capacidad predictiva a escala estacional, consecuencia de la baja predecibilidad en las latitudes medias.

Sin embargo, siendo la predicción estacional un campo en pleno desarrollo, AEMET está mejorando estas predicciones por medio de varias líneas de investigación. La concreción de estas mejoras y su aplicación al proyecto produciría una mejora significativa del producto estacional del proyecto.

\section{CONCLUSIÓN}

Se ha desarrollado un servicio climático adecuado para ayudar a las necesidades de los usuarios finales del sector agrario. Este servicio hace uso de predicciones a corto/medio plazo que tienen una precisión adecuada para esta aplicación, y de predicciones estacionales regionalizadas.

\section{AGRADECIMIENTOS}

Los autores agradecen la inestimable ayuda técnica y de documentación del Departamento de Desarrollo y Aplicaciones (Javier Calvo, María Díez, Gema Morales...), del Departamento de Producción (Alberto Fernández Matía, Carlos Santos, Área de Explotación...), etc.

\section{REFERENCIAS}

Piani, C., Haerter, J. O. y Coppla, E., 2010. Statistical bias correction for daily precipitation in regional climate models over Europe. Theor. Appl. Climatol., 99: 187-192.

Proyecto MOSES, 2017. Presentación. Jornadas sobre Innovación en gestión de regadíos mediante redes agroclimáticas, imágenes de satélite y sistemas de información geográfica. San Fernando de Henares (Madrid). 
Centro Nacional de Tecnología de Regadíos, Subdirección General de Regadíos y Economía del Agua (MAPAMA). 19 de diciembre de 2017.

http://www.mapama.gob.es/fr/desarrollo-rural/temas/centro-nacional-tecnologia-regadios/formacion-difusiontecnologica/documentacion/

Sánchez García, E., Voces Aboy, J. y Rodríguez Camino, E., 2017. Verification of seasonal forecasts over Europe and Northern Africa. En revisión. Se publicará en: http://medcof.aemet.es/index.php/models-skillover-mediterranean.

Serrano de la Torre, A. Á., Mestre Guillén, I., Rodríguez Camino, E. y López Díaz, J. A., 2017. Servicios climáticos para la gestión del regadío. 10. ${ }^{\circ}$ Simpósio de Meteorologia e Geofísica da APMG. http://hdl.handle.net/20.500.11765/7328. 